
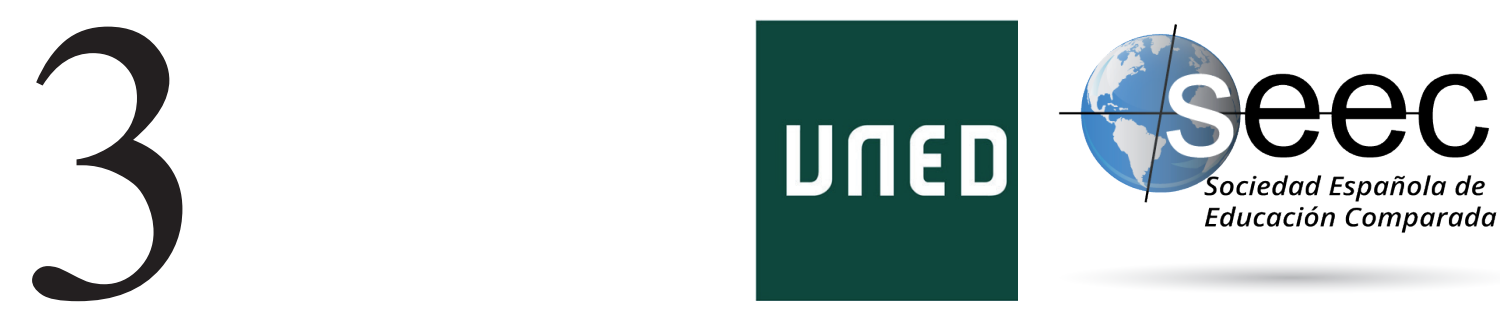

\title{
La Historia de la Educación tras Bolonia: ¿hacia la extinción?**
}

\author{
History of Education after Bologna \\ process: towards extinction?
}

\author{
Antonio Fco. Canales Serrano**; Yasmina Álvarez \\ González $^{* * *}$ y María José Tacoronte Domínguez ${ }^{* * * *}$
}

DOI: $10.5944 /$ reec.29.2017.18632

\section{Recibido: 12 de marzo de 2017 Aceptado: 18 de abril de 2017}

\begin{abstract}
* Este artículo se ha realizado en el marco del Proyecto de I+D «La frontera entre ciencia y política y la ciencia en la frontera: la ciencia española, 1907-1975», FFI2015-64529-P (MINECO/FEDER).

* Antonio Fco. Canales Serrano: es Doctor en Historia y Profesor Titular de Historia de la Educación en la Universidad de La Laguna. Su investigación se ha centrado en la historia del régimen de Franco con especial atención a la educación, el género y la ciencia. Es autor de libros como Las otras derechas (Marcial Pons, Madrid 2006) y coeditor de La larga noche de la educación española (Biblioteca Nueva, Madrid 2015), Science Policies and Twentieth-Century Dictatorships (Routledge, London 2015) y Women's Education in the Mediterranean World: Historical Perspectives (1840-1970) (Roma, Aracne, 2017). Ha publicado en las principales revistas de su campo como Paedagogica Historica, History of Education, Gender and Education, Bordón, Educación XX1, Ayer, Historia Social e Historia Contemporánea. Es investigador principal del proyecto nacional de investigación La frontera entre ciencia y política y la ciencia en la frontera: la ciencia española, 1907-1975, FFI2015-64529-P. Es miembro de la Junta Directiva de la Sociedad Española de Historia de la Educación y secretario de su revista Historia y Memoria de la Educación. Datos de contacto: OrCID: http://orcid.org/oooo-0002-7035-1194. E-mail: acanales@ull.edu.es

*** Yasmina Álvarez GonZÁlez: es profesora de Teoría e Historia de la Educación en la Universidad de La Laguna. Es miembro del equipo de trabajo del proyecto nacional de investigación La frontera entre ciencia y política y la ciencia en la frontera: la ciencia española, 1907-1975, FFI2015-64529-P. Sus líneas de investigación se centran en la historia de la Pedagogía como disciplina académica bajo el primer franquismo y en la perspectiva de género. En este sentido ha publicado «La concepción de $\mathrm{M}^{\mathrm{a}}$ Ángeles Galino sobre la mujer», Cuadernos del Ateneo de La Laguna, 32 (2014) y «Las mujeres en la pedagogía franquista. Aproximación a la presencia femenina en el Instituto San José de Calasanz del CSIC». Memorias del IX Congreso Iberoamericano de Ciencia, Tecnología y Género. Madrid. Organización de Estados Iberoamericanos, 2012. Datos de contacto: E-mail: yalvarez@ull.edu.es
\end{abstract}

**** Ma José TACoronte Domínguez: es licenciada en Filosofía por la Universidad de La Laguna. Actualmente es profesora de Teoría e Historia de la Educación en la Universidad de La Laguna. Sus líneas de investigación se han centrado en la filosofía de la ciencia, los estudios políticos y de género en el ámbito científico, la historia de la ciencia y de la educación. Ha sido investigadora en varios proyectos de investigación, en la actualidad participa en el proyecto de I+D+i La frontera entre ciencia y política y la ciencia en la frontera: La ciencia española 1907-1975. FFI2015-64529-P. Es autora, entre otros trabajos, de «G. Marañón y R. Novoa: Del imperativo natural al imperativo social». Análisis. Revista de investigación filosófica.3 - 1, Zaragoza (España): Universidad de Zaragoza, 2016 y «La biopolítica española: el caso de Roberto Novoa Santos» en Gómez, A. y Canales, A. Fco (2013): Estudios políticos de la ciencia. Políticas y desarrollo científico en el siglo $X X$. Plaza y Valdés Editores. Datos de contacto: E-mail: mjtacoro@ull.edu.es 


\title{
Resumen
}

Este artículo compara la presencia de la historia de la educación en las extintas licenciaturas y en los nuevos grados de Pedagogía establecidos tras el proceso de Bolonia. Para ello analiza la situación de la historia de la educación en los planes de estudios de Pedagogía en las universidades públicas españolas desde diferentes variables como la carga obligatoria y optativa, la ubicación en los cursos y los criterios para la configuración de asignaturas optativas. La comparación arroja como resultado la drástica disminución de los contenidos de historia de la educación en los planes de estudio de Pedagogía, con una reducción a la mitad en el caso de la obligatoriedad y a un tercio en la optatividad. La historia de la educación ha quedado circunscrita a una asignatura de primer ciclo introductoria y secundaria que dos tercios de las universidades complementan con una segunda asignatura. Se completa así el proceso iniciado en los setenta de progresiva erosión de la tradicional posición central de la historia de la educación en la formación de los futuros profesionales de la Pedagogía. Un paso más supondría la práctica desaparición del campo disciplinar.

Palabras clave: Historia de la educación; Convergencia Europea; Proceso de Bolonia; Pedagogía; España

\begin{abstract}
This article compares the weight of history of education in the old Spanish degrees of Pedagogy and in the new ones established after the Bologna Process. In order to do this, it analyzes the situation of history of education courses in the curriculum of Pedagogy in Spanish public universities from different variables such as the compulsory or elective character, the location in the years and the criteria for the configuration of elective courses. The comparison shows a drastic reduction of the contents related to History of Education in the curricula of Pedagogy, with a reduction in half in the case of compulsory courses and to one-third in elective one. History of Education has been limited to a first year course, configured as an introductory and secondary course, which two thirds of the universities complement with a second course. This completes the process begun in the seventies of progressive erosion of the traditional central position of history of education in the education of the future professionals of pedagogy. A further step would be the practical disappearance of the disciplinary field.
\end{abstract}

Key Words: History of Education; European Higher Education Area (EHEA); Bologna Process; Pedagogy; Spain 


\section{Introducción}

La historia de la educación fue durante muchas décadas uno de los pilares de la formación de los profesionales de la educación, maestros en primer lugar y posteriormente también pedagogos (RICHARDSON, 2000:19; DEPAEPE, 2007:17). No resulta extraño, pues, que el campo se definiera en estrecha relación con la formación de docentes desde el siglo xix hasta el último tercio del siglo xx. Así, en España la historia de la educación figuraba entre las ocho cátedras sobre las que se constituía el primer título universitario de Pedagogía en 1932 (MAINER, 2009: 266). En 1944, bajo un régimen diametralmente opuesto, la historia de la educación mantenía una posición central como asignatura obligatoria en tres de los seis cuatrimestres de la especialización (BOE, 4/8/1944). Esta centralidad en la formación de maestros y pedagogos comenzó a verse cuestionada en los años setenta. En las últimas décadas, tanto en España como a escala internacional, la historia de la educación ha ido perdiendo su antigua condición de saber fundamental en la formación de estos colectivos para perfilarse progresivamente como una materia introductoria y secundaria (MCCULLOCH, 2011).

Esta progresiva pérdida de centralidad de la historia de la educación ha dado lugar a una notable reflexión tanto a escala nacional (GUEREÑA, RUIZ BERRIO, TIANA, 2010; SANCHIDRIÁN, 2013 y 2016; VIÑAO, 2008 y 2016) como internacional (FUCHS, 2010; CASPARD and ROGERS, 2012; OSTERWALDER, 2012; FITZGERALD, 2013; LE CAM, 2013). Se ha constituido además un grupo de trabajo en el seno de la International Standing Conference of History of Education (ISCHE) denominado Mapping the Discipline History of Education, bajo la dirección de Eckhardt Fuchs, Rita Hofstetter, Emmanuelle Picard y Solenn Huitric. A través de los simposios internacionales celebrados desde 2014 en Londres, Estambul y Chicago, este grupo viene recopilando información con el fin de realizar una cartografía internacional de la situación institucional de la historia de la educación. (HOFSTETTER, FONTAINE, HUITRIC and PICARD, 2014; ISCHE).

Desarrollando esta línea internacional de trabajo, el presente artículo pretende dar cuenta de este proceso de marginalización de la historia de la educación en España a través de un estudio comparativo de la situación de la disciplina antes y después de la última reforma de los planes de estudios realizada en el marco del proceso de Bolonia, que sustituyó las tradicionales licenciaturas por los nuevos grados. Para ello se han tomado en consideración los planes de estudios de las 18 universidades públicas españolas que ofrecían la licenciatura de Pedagogía con anterioridad a la reforma (dos de ellas sólo como titulación de segundo ciclo), que son las mismas que en la actualidad ofrecen los grados. La comparación, pues, se basa en la totalidad de los títulos ofrecidos por universidades públicas y no incluye las privadas. Para los planes de estudio se ha tomado como base el Boletín Oficial del Estado y se ha recurrido puntualmente a las guías docentes de las asignaturas en los casos en que los contenidos no quedan claros en el título o se presentan combinados. La presencia de la historia de la educación en estos títulos se analiza en función de cuatro variables básicas: el número de créditos, la obligatoriedad - optatividad, la ubicación en los cursos y los criterios para la creación de asignaturas optativas. 


\section{Las extintas licenciaturas}

En las extintas licenciaturas de Pedagogía, la carga media de las asignaturas de historia de la educación superaba los 15 créditos obligatorios (troncales y obligatorios de universidad), cifra que venía a suponer un $5 \%$ de la carga total del título. Por encima de esta carga se situaban siete universidades, entre las que destacaban Valencia con 21 y La Laguna con 19,5 (7 \% y 6 \% del título respectivamente); por debajo de la media se ubicaban sólo tres universidades, de las cuales dos, Santiago de Compostela y la Autónoma de Barcelona, se alejaban notablemente de la pauta común al ofrecer una carga muy inferior a la media, 9 y 8 créditos respectivamente.

Tabla 1. Créditos de historia de la educación en las licenciaturas de Pedagogía extinguidas.

Fuente. Elaboración propia a partir de información oficial.

\begin{tabular}{|l|c|c|}
\hline \multicolumn{1}{|c|}{ Universidad $^{\S}$} & Oblig. & $\begin{array}{c}\text { Totales: } \\
\text { Obl. + Opt. }\end{array}$ \\
\hline UV & 21 & 55,5 \\
\hline ULL & 19,5 & 33 \\
\hline UB & 18 & 48 \\
\hline UOV & 18 & 27 \\
\hline UCM & 18 & 24 \\
\hline US & 17 & 26 \\
\hline UMA & 16,5 & 34,5 \\
\hline UGR & 15 & 33 \\
\hline UM & 15 & 28,5 \\
\hline UPV & 15 & 27 \\
\hline UIB & 15 & 27 \\
\hline UNED & 15 & 25 \\
\hline USAL & 15 & 24 \\
\hline URV & 13,5 & 31,5 \\
\hline USC & 9 & 13,5 \\
\hline UAB & 8 & 15 \\
\hline UdG* & 6 & 15 \\
\hline UBU* & 0 & 24 \\
\hline MEDIA & \multicolumn{2}{|l}{} \\
\hline \&Véase listado de abreviaturas en el Anexo I \\
\hline * Titulación de segundo & 30,09 \\
\hline \& Sólo titulaciones completas & \\
\hline
\end{tabular}

La toma en consideración de la optatividad incrementa notablemente tanto la carga total como la variabilidad de casos. En general, la carga media incluyendo las asignaturas optativas se situaba en 30 créditos, con el grueso de las universidades situadas entre 24 y 33. Fuera de este rango descollaban Valencia con 55,5 créditos y Barcelona, que con 48 disparaba su carga con respecto a la obligatoriedad. En el otro extremo, las universidades de Santiago de Compostela y Autónoma de Barcelona repetían como las titulaciones con menor presencia de la historia de la educación, igual o incluso menor que en las universidades que sólo ofrecían la titulación en segundo ciclo. 
Tabla 2. Universidades con sólo una asignatura obligatoria. Fuente. Elaboración propia a partir de información oficial.

\begin{tabular}{|c|l|c|c|c|c|}
\hline Univ. & \multicolumn{1}{|c|}{ Asignatura } & Tipo & Curso & Créd. & Total \\
\hline \multirow{2}{*}{ UAB } & Historia de la Educación & TR & I & 8 & 8 \\
\cline { 2 - 5 } & Historia de la Educación Social & OPT & 2 ciclo & 4 & \multirow{2}{*}{15} \\
\cline { 2 - 6 } & Historia de la Educación en Cataluña & OPT & 2 ciclo & 3 & \\
\hline \multirow{2}{*}{ USC } & Historia de la Educación & TR & I ciclo & 9 & 9 \\
\cline { 2 - 6 } 200 I & Educación y Sociedad en Galicia & OPT & I ciclo & 4,5 & 13,5 \\
\hline
\end{tabular}

Si se atiende al número de asignaturas en que se concretaba la obligatoriedad, las universidades que menos créditos incluían, Autónoma de Barcelona y Santiago de Compostela, constituyen un grupo diferenciado con una única asignatura. La opción en ambas era cumplir únicamente con la troncalidad establecida por el ministerio, de manera estricta en el caso de la Autónoma de Barcelona. Sin embargo, esta no fue la fórmula más común como muestran las siguientes tablas.

Tabla 3. Universidades con cuatro asignaturas o más de historia de la educación. Fuente. Elaboración propia a partir de información oficial.

\begin{tabular}{|c|c|c|c|c|c|}
\hline Univ. & Asignatura & Tipo & Curso & Créd. & Total \\
\hline \multirow{2}{*}{$\begin{array}{l}\text { URV } \\
1993\end{array}$} & Historia de la Educación & $T R$ & 1 & 9 & \multirow[b]{2}{*}{13,5} \\
\hline & $\begin{array}{l}\text { Teoría e Instituciones Contemporáneas } \\
\text { de la Educación }\end{array}$ & OBL & I & 4,5 & \\
\hline \multirow{4}{*}{2007} & $\begin{array}{l}\text { Teoría e Historia de la Educación de } \\
\text { Género }\end{array}$ & OPT & & 4,5 & \multirow{4}{*}{31,5} \\
\hline & Historia de la Pedagogía Catalana & OPT & & 4,5 & \\
\hline & Teoría e Historia de la Educación Juvenil & OPT & & 4,5 & \\
\hline & $\begin{array}{l}\text { Teoría e Historia de las Instituciones } \\
\text { Educativas Europeas }\end{array}$ & OPT & & 4,5 & \\
\hline \multirow{4}{*}{$\begin{array}{l}\text { USAL } \\
2000\end{array}$} & Historia de la Educación & TR & I & 9 & \multirow{2}{*}{15} \\
\hline & Historia de la Educación Española & TR & 3 & 6 & \\
\hline & Historia de la Educación en América & OPT & 4 & 4,5 & \multirow{4}{*}{15} \\
\hline & $\begin{array}{l}\text { Historia de la Educación en Castilla- } \\
\text { León }\end{array}$ & OPT & 4 & 4,5 & \\
\hline \multirow{4}{*}{$\begin{array}{c}\text { UNED } \\
1999\end{array}$} & Historia de la Educación & TR & 1 & 10 & \\
\hline & Historia de la Educación Española & OBL & 2 & 5 & \\
\hline & Historia del Currículum & OPT & & 5 & \multirow{2}{*}{25} \\
\hline & Historia de la Infancia & OPT & & 5 & \\
\hline \multirow{4}{*}{$\begin{array}{c}\text { UIB } \\
1997\end{array}$} & Historia de la Educación & TR & 1 & 9 & \multirow[b]{2}{*}{15} \\
\hline & $\begin{array}{l}\text { Historia de la Educación Catalana } \\
\text { Contemporánea }\end{array}$ & OBL & 3 & 6 & \\
\hline & Historia de la Educación Social & OPT & & 6 & \multirow[b]{2}{*}{27} \\
\hline & $\begin{array}{l}\text { Historia de la Escolarización y la } \\
\text { Instrucción }\end{array}$ & OPT & & 6 & \\
\hline
\end{tabular}


Tabla 3. (Continuación)

\begin{tabular}{|c|c|c|c|c|c|}
\hline Univ. & Asignatura & Tipo & Curso & Créd. & Total \\
\hline \multirow{4}{*}{$\begin{array}{l}\text { UPV } \\
1999\end{array}$} & Historia de la Educación & TR & 2 & 9 & \multirow{2}{*}{15} \\
\hline & Historia de la Educación en el País Vasco & OBL & 4 & 6 & \\
\hline & $\begin{array}{l}\text { Curso Monográfico de Historia de la } \\
\text { Educación }\end{array}$ & OPT & 2 ciclo & 6 & \multirow{4}{*}{15} \\
\hline & Historia de la Escuela & OPT & 2 ciclo & 6 & \\
\hline \multirow{5}{*}{$\begin{array}{l}\text { UM } \\
2001\end{array}$} & Historia de la Educación & TR & 1 & 8 & \\
\hline & Historia de la Educación Española & OBL & 2 & 7 & \\
\hline & Historia Social de la Educación & OPT & I ciclo & 4,5 & \multirow{3}{*}{28,5} \\
\hline & Historia del Currículum & OPT & 2 ciclo & 4,5 & \\
\hline & Historia de la Educación de la Mujer & OPT & 2 ciclo & 4,5 & \\
\hline \multirow{5}{*}{$\begin{array}{l}\text { UGR } \\
2001\end{array}$} & Historia de la Educación & TR & 1 & 9 & \multirow{2}{*}{15} \\
\hline & Historia del Currículum & OBL & 2 & 6 & \\
\hline & Historia de la Educación de las Mujeres & OPT & & 6 & \multirow{5}{*}{16,5} \\
\hline & $\begin{array}{l}\text { Historia de la Educación de la Infancia y } \\
\text { su Escolarización }\end{array}$ & OPT & & 6 & \\
\hline & Investigación Histórica en Educación & OPT & & 6 & \\
\hline \multirow{6}{*}{$\begin{array}{l}\text { UMA } \\
1999\end{array}$} & Historia de la Educación & TR & 2 & 9 & \\
\hline & Historia de la Educación en España & OBL & 3 & 7,5 & \\
\hline & Historia del Currículum & OPT & & 4,5 & \multirow{6}{*}{34,5} \\
\hline & Historia de la Educación en Andalucía & OPT & & 4,5 & \\
\hline & $\begin{array}{l}\text { Infancia y Educación Infantil en la } \\
\text { Historia }\end{array}$ & OPT & & 4,5 & \\
\hline & Historia de la Profesión Docente & OPT & & 4,5 & \\
\hline \multirow{4}{*}{1998} & Historia de la Educación & TR & I & 9 & \\
\hline & Historia de la Educación en España & OBL & 2 & 8 & \\
\hline & Historia de la Educación en Andalucía & OPT & 1 & 4,5 & \multirow[b]{2}{*}{26} \\
\hline & $\begin{array}{l}\text { Historia y Desarrollo Socioeducativo de } \\
\text { las Mujeres }\end{array}$ & OPT & 4 & 4,5 & \\
\hline \multirow{2}{*}{ UCM } & Historia de la Educación & TR & 2 & 9 & \multirow{2}{*}{18} \\
\hline & Historia de la Educación en España & OBL & 3 & 9 & \\
\hline 2000 & $\begin{array}{l}\text { Historia de la Educación en la } \\
\text { Comunidad de Madrid }\end{array}$ & OPT & 5 & 6 & 24 \\
\hline \multirow{4}{*}{$\begin{array}{l}\text { UOV } \\
2000\end{array}$} & Historia de la Educación & TR & 2 & 9 & \multirow{2}{*}{18} \\
\hline & Historia de la Educación en España & OBL & 3 & 9 & \\
\hline & Historia del Currículum & OPT & & 4,5 & \multirow[b]{2}{*}{27} \\
\hline & $\begin{array}{l}\text { La Educación Social en la Época } \\
\text { Moderna y Contemporánea }\end{array}$ & OPT & & 4,5 & \\
\hline \multirow{4}{*}{$\begin{array}{l}\text { ULL } \\
2000\end{array}$} & Historia de la Educación & TR & 1 & 9 & 19,5 \\
\hline & $\begin{array}{l}\text { Historia de la Educación Moderna y } \\
\text { Contemporánea }\end{array}$ & OBL & 2 & 10,5 & \multirow[b]{3}{*}{33} \\
\hline & Historia de la Educación en Canarias & OPT & 2 ciclo & 6 & \\
\hline & $\begin{array}{l}\text { Pedagogía Contemporánea en España y } \\
\text { América Latina }\end{array}$ & OPT & 2 ciclo & 7,5 & \\
\hline
\end{tabular}


Tabla 4. Universidades con tres asignaturas de historia de la educación. Fuente. Elaboración propia a partir de información oficial.

\begin{tabular}{|c|c|c|c|c|c|}
\hline Univ. & Asignatura & Tipo & Curso & Créd. & Total \\
\hline \multirow{5}{*}{$\begin{array}{c}\text { UB } \\
2000\end{array}$} & $\begin{array}{l}\text { Historia de la Educación hasta la Rev. } \\
\text { Francesa }\end{array}$ & $\mathrm{TR}$ & 1 & 6 & \multirow{3}{*}{18} \\
\hline & $\begin{array}{l}\text { Historia de la Educación } \\
\text { Contemporánea }\end{array}$ & TR & 1 & 6 & \\
\hline & $\begin{array}{l}\text { Historia de la Educación en la Cataluña } \\
\text { Contemp. }\end{array}$ & OBL & 2 ciclo & 6 & \\
\hline & Historia de la Infancia & OPT & I ciclo & 6 & \multirow[b]{2}{*}{48} \\
\hline & Historias Sectoriales de la Educación & OPT & 2 ciclo & 24 & \\
\hline \multirow{10}{*}{$\begin{array}{c}\text { UV } \\
2000\end{array}$} & Historia de la Educación & TR & $\mathrm{I}$ & 9 & \multirow{3}{*}{21} \\
\hline & Historia de la Escuela & OBL & 3 & 6 & \\
\hline & Historia de la Educación en España & OBL & 4 & 6 & \\
\hline & Historia de la Educación Especial & OPT & I ciclo & 6 & \multirow{7}{*}{55,5} \\
\hline & $\begin{array}{l}\text { Historia de la Educación Popular y de } \\
\text { Adultos }\end{array}$ & OPT & I ciclo & 6 & \\
\hline & $\begin{array}{l}\text { Historia de los Sistemas Educativos } \\
\text { Europeos }\end{array}$ & OPT & I ciclo & 4,5 & \\
\hline & Historia de la Educación de las Mujeres & OPT & 2 ciclo & 4,5 & \\
\hline & Historia de la Educación Valenciana & OPT & 2 ciclo & 4,5 & \\
\hline & $\begin{array}{l}\text { Historia de la Educación Social en } \\
\text { España }\end{array}$ & OPT & 2 ciclo & 4,5 & \\
\hline & Historia Política de la Educación & OPT & 2 ciclo & 4,5 & \\
\hline
\end{tabular}

Tabla 5. Universidades con titulación de segundo ciclo. Fuente. Elaboración propia a partir de información oficial.

\begin{tabular}{|c|c|c|c|c|c|}
\hline Univ. & Asignatura & Tipo & Curso & Créd. & Total \\
\hline \multirow{4}{*}{$\begin{array}{l}\text { UBU } \\
1998\end{array}$} & Historia de la Educación en España & OPT & 5 & 6 & \multirow{4}{*}{24} \\
\hline & $\begin{array}{l}\text { Análisis Histórico de las Reformas } \\
\text { Educativas }\end{array}$ & OPT & 4 & 6 & \\
\hline & Historia de la Infancia y de la Escuela & OPT & 5 & 6 & \\
\hline & $\begin{array}{l}\text { Evolución Histórica de la Educación } \\
\text { Especial en Europa }\end{array}$ & OPT & 5 & 6 & \\
\hline \multirow{3}{*}{$\begin{array}{l}\text { UdG } \\
2000\end{array}$} & Historia de la Educación & $\mathrm{OBL}$ & 3 & 6 & 6 \\
\hline & $\begin{array}{l}\text { Evolución Histórica de los Movimien- } \\
\text { tos Educativos y Sociales Contem- } \\
\text { poráneos }\end{array}$ & OPT & 2 ciclo & 4,5 & \multirow[t]{2}{*}{15} \\
\hline & Historia de la Pedagogía en Cataluña & OPT & 2 ciclo & 4,5 & \\
\hline
\end{tabular}

La inmensa mayoría de las universidades (12 sobre 16 con titulación completa) optó por completar la troncalidad establecida por el ministerio con una segunda asignatura obligatoria de universidad. El criterio mayoritario para esta división en dos asignaturas era el geográfico. Nueve universidades añadían a la genérica Historia de la Educación 
una asignatura específica para un ámbito geográfico concreto, España en siete de ellas, el País Vasco en esta universidad y Cataluña en la Universitat de les Illes Balears. Sólo tres universidades de este grupo con dos asignaturas utilizaron un criterio alternativo al geográfico. La Universidad de la Laguna optó por un criterio cronológico introduciendo una Historia de la Educación Moderna y Contemporánea; Granada utilizó un criterio temático para crear una Historia del Currículum y en la Rovira i Virgili encontramos ya la combinación de los contenidos históricos con otros en una Teoría e Instituciones Educativas Contemporáneas, una combinación restringida al área de Teoría e Historia de la Educación.

Finalmente, dos universidades planteaban tres asignaturas obligatorias de historia de la educación a partir de combinaciones específicas de los criterios señalados con anterioridad. La Universitat de Barcelona utilizaba el criterio cronológico utilizado también por la Universidad de La Laguna (en este caso restringido a la Edad Contemporánea) y lo completaba con el criterio geográfico para incluir una Historia de la Educación en la Cataluña Contemporánea. La Universitat de València, por su parte, combinaba este criterio geográfico con el criterio sectorial de la Universidad de Granada para ofertar una Historia de la Escuela, además de una Historia de la Educación en España.

La toma en consideración de la optatividad ampliaba notablemente el abanico de asignaturas. El criterio más común de delimitación de optativas era el geográfico aplicado a la propia comunidad autónoma. Aparecían, así, dos historias de Cataluña optativas, que se sumaban a las dos obligatorias, dos de Andalucía y una de Canarias, Castilla-León, Galicia, Madrid y Valencia. Se creaba así una significativa combinación de territorialidad y obligatoriedad/optatividad de la que da cuenta el diagrama 1.

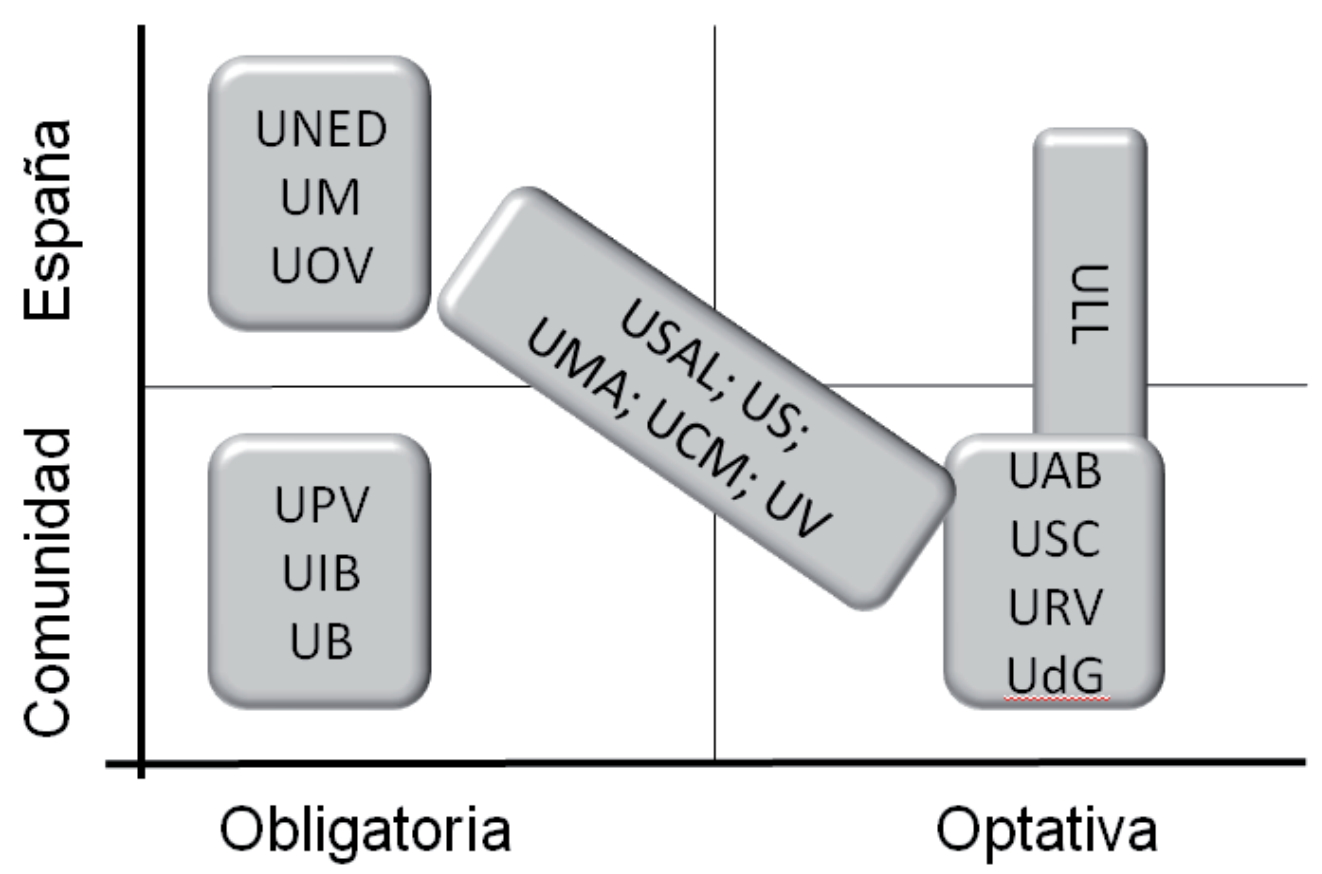

Diagrama 1. Asignaturas con concreción geográfica por ámbito de referencia y carácter obligatorio u optativo. Fuente. Elaboración propia a partir de información oficial. 
Tres universidades (UNED, Murcia y Oviedo) ofertaban como única concreción territorial una historia de la educación en España de carácter obligatorio. En el otro extremo, las universidades de las comunidades con movimientos nacionalistas propios (o en su ámbito de influencia cultural como Baleares) incluían únicamente la historia de la educación en la comunidad respectiva, ya fuera en la modalidad obligatoria como en el País Vasco, Barcelona e Illes Balears o como optativa en la Rovira i Virgili, Autònoma de Barcelona y Santiago de Compostela. Sólo seis universidades parecían considerar que las concreciones española y autonómica no eran excluyentes y combinaban en su oferta una historia de España obligatoria y una historia de la comunidad optativa (Complutense de Madrid, Málaga, Murcia, Sevilla, Salamanca y Valencia). La Laguna presentaba un modelo particular que fundía España con Latinoamérica en la misma asignatura optativa, en paralelo con Canarias. Salamanca, por su parte, desarrollaba esta dimensión iberoamericana con una Historia de la Educación monográficamente dedicada a América. Así, pues, los contenidos americanistas sólo se contemplaban en los rótulos de las asignaturas de La Laguna y Salamanca. Finalmente, la Rovira i Virgili y Valencia eran la únicas en incluir una asignatura con Europa como marco.

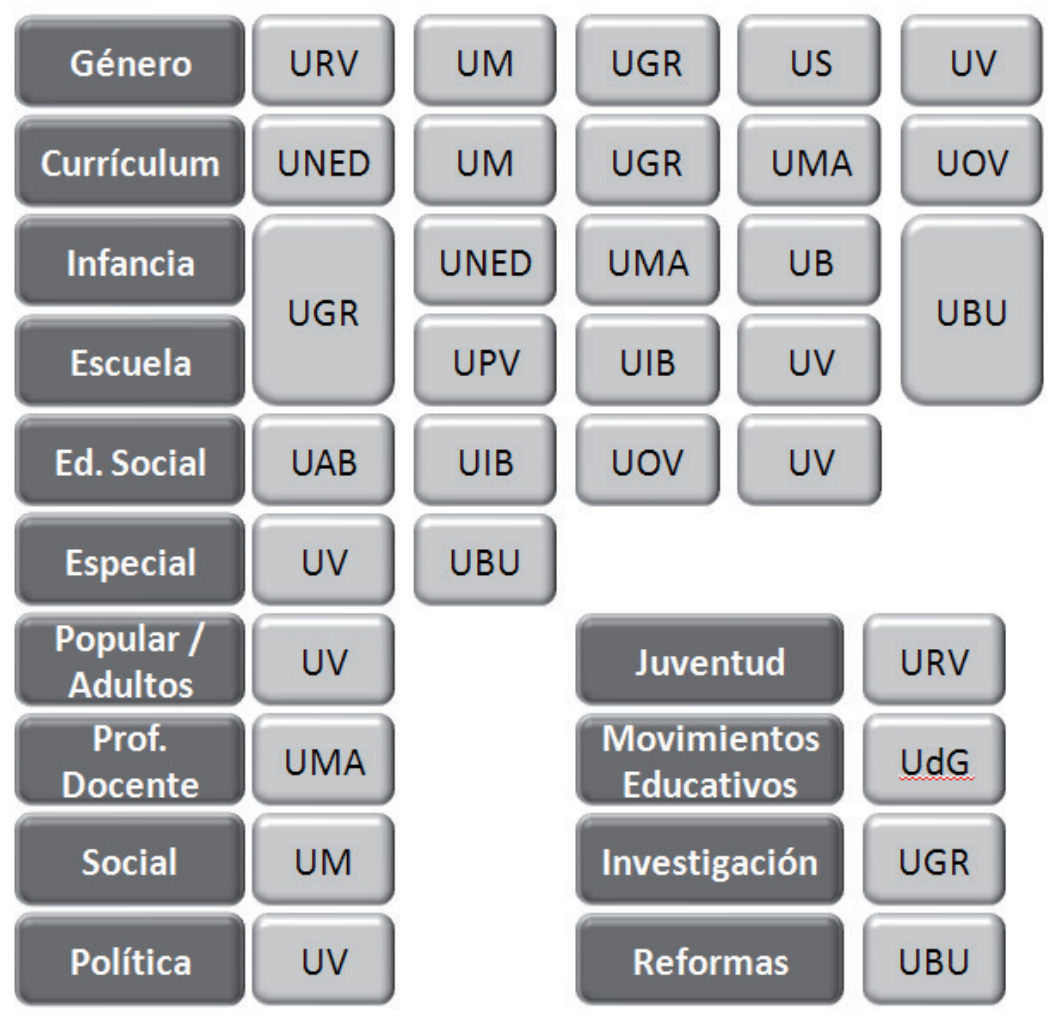

Diagrama 2. Asignaturas sin concreción geográfica. Fuente. Elaboración propia a partir de información oficial.

Este acuerdo general en el criterio geográfico no se reproducía en los criterios utilizados para delimitar el resto de las asignaturas optativas, que eran muy variados y claramente condicionados por los créditos ofrecidos por cada universidad. Como muestra el diagrama 2, los más comunes entre ellos eran la perspectiva de género y la Historia 
del Currículum, optativas ofertadas en cinco universidades. Granada, Murcia, Rovira i Virgili, Sevilla y Valencia ofertaban bajo diferentes rótulos contenidos de Historia de la Educación de las Mujeres y Granada, Málaga, Murcia, Oviedo y la UNED una Historia del Currículum. A continuación se situaban las Historias de la Infancia (Barcelona, Málaga y UNED) y de la Escuela o la Escolarización (Illes Balears, País Vasco y Valencia), opciones que Granada y Burgos combinaban en una única asignatura. Un poco por debajo en popularidad se situaba la Historia de la Educación Social (Autònoma de Barcelona, Illes Balears, Oviedo y Valencia). Fuera de este repertorio quedaban la Historia de la Educación Especial, ofertada únicamente por dos universidades (Valencia y Burgos) y un conjunto de optativas únicas en cada universidad. Entre ellas se encontraban las historias relacionas con grupos de edad como la juventud (Rovira i Virgili) y adultos, unidos a la educación popular (Valencia), con colectivos profesionales como la profesión docente (Málaga), los movimientos educativos (Girona) o las reformas (Burgos). Finalmente, Murcia y Valencia optaban por subrayar una perspectiva histórica concreta con una historia social de la educación en el primer caso y una historia política en el segundo. Granada, por su parte, se singularizaba con una asignatura teórico-metodológica sobre investigación histórica en educación.

\section{Recapitulación:}

Dos universidades españolas, el 12,5 \%, se ajustaban al mínimo legal de ocho créditos de Historia de la Educación establecido por el ministerio para los planes de estudios de Pedagogía (Santiago de Compostela [9] y Autónoma de Barcelona).

Seis universidades, el $37,5 \%$, ofrecían 15 créditos troncales y obligatorios (Illes Balears, Granada, Murcia, País Vasco, Salamanca y UNED).

El grueso de las universidades (12 - 75 \%) situaba su oferta troncal y obligatoria entre los 13,5 créditos y los 18, con la Rovira i Virgili en la posición inferior y Barcelona, Complutense de Madrid y Sevilla en la superior.

Sólo dos universidades, el 12,5 \%, ofrecían más de 18 créditos: La Laguna con 19,5 y Valencia con 21.

Doce universidades (el 75 \%) contemplaba una segunda asignatura obligatoria, mayoritariamente una historia de la educación en España, y dos ellas, Barcelona y Valencia, introducían tres.

La oferta de contenidos de Historia de la Educación se incrementaba notablemente gracias a la optatividad hasta una media de 30 créditos. 12 universidades (75 \%) situaban su oferta global entre los 24 créditos y los 34,5. Por debajo, destacaban de nuevo la Universitat Autònoma de Barcelona y Santiago de Compostela con apenas 15 y 13,5 créditos respectivamente. Por encima, descollaban Barcelona con 48 y Valencia con 55,5.

La introducción de Historias de la Educación de la comunidad autónoma respectiva era el criterio más común para la creación de asignaturas optativas. Entre obligatorias y optativas, sólo seis universidades (37,5 \%) ofertaban a la vez Historias de España y la comunidad autónoma, mientras que tres $(18,75 \%)$ ofertaban sólo una Historia de España y seis (37\%) sólo la historia de la comunidad. 
Tras las historias acotadas geográficamente, las optativas más comunes eran:

- Historia de la Educación de las Mujeres (Granada, Murcia, Rovira i Virgili, Sevilla y Valencia)

- Historia del Currículo (Granada, Málaga, Murcia, Oviedo y UNED)

- Historia de la Educación Social (Autònoma de Barcelona, Illes Balears Oviedo, Valencia)

- Historia de la Infancias (Barcelona, Málaga y UNED)

- Historia de la Escuelas o de la Escolarización (Illes Balears, País Vasco y Valencia)

La Universitat de Valéncia se situaba en la cabeza de las universidades españolas tanto por número de créditos de Historia de la Educación ofrecidos $(55,5)$ como por asignaturas: tres troncales/obligatorias y siete optativas.

\section{Los nuevos grados ${ }^{1}$}

Tabla 6. Créditos de Historia de la Educación en los grados de Pedagogía. Fuente. Elaboración propia a partir de información oficial.

\begin{tabular}{|l|c|c|}
\hline \multicolumn{1}{|c|}{ Universidad $\S$} & Obl. & Totales \\
\hline UV & 12 & 2 I \\
\hline UCM & 12 & 18 \\
\hline UGR & 12 & 18 \\
\hline US & 12 & 18 \\
\hline ULL & 12 & 12 \\
\hline UMA & 12 & 12 \\
\hline UM & 12 & 12 \\
\hline UOV & 12 & 12 \\
\hline UNED & 12 & 12 \\
\hline UB & 6 & 12 \\
\hline UPV & 6 & 12 \\
\hline USAL & 6 & 12 \\
\hline UdG & $\mathbf{6}$ & $\mathbf{9}$ \\
\hline UIB & 6 & 6 \\
\hline USC & 6 & 6 \\
\hline UAB & 4 & 4 \\
\hline UBU & $\mathbf{3}$ & $\mathbf{2}$ \\
\hline URV & & $?$ \\
\hline MEDIA & & 11 \\
\hline §Véase listado de abreviaturas en el Anexo I & \\
\hline
\end{tabular}

1 Para el proceso de elaboración de los nuevos grados de Pedagogía y su contextualización, véase GRANA, 2012. Para su análisis véase, además del anterior, ÁLVAREZ, 2012. 
La carga lectiva media obligatoria dedicada a la Historia de la Educación en los nuevos grados de Pedagogía de las universidades públicas españolas ronda los ocho créditos. No obstante, como muestra la tabla 6 , este dígito no es más que un efecto aritmético, pues ninguna universidad se acerca a esa carga. La mitad de las universidades se sitúa notalmente por encima con una oferta de 12 créditos obligatorios ( $5 \%$ de la carga del título), mientras que la otra mitad ofrece seis créditos o incluso menos (2,5\%). Esta carga se traduce en dos asignaturas obligatorias de seis créditos en el primer bloque y en una, o menos, en el segundo.

La inclusión de la optatividad amplía la variabilidad, aunque no tanto como en las licenciaturas. La media de oferta total se sitúa en los 11 créditos, por encima de la cual se sitúan dos tercios de las universidades: ocho con 12 créditos, tres con 18 (Complutense de Madrid, Granada y Sevilla) y con 21 la Universitat de València, que es la universidad española que más peso confiere a la historia de la educación en su grado.

Por debajo de la media, se encuentran cinco universidades. Dos de ellas, (Illes Balears y Santiago de Compostela) otorgan prácticamente la mitad de la media a la Historia de la Educación con apenas seis créditos y la de Girona un poco más con nueve. En las posiciones finales, tres universidades se sitúan incluso por debajo de los seis créditos que suponen una asignatura e integran la Historia de la Educación en asignaturas más amplias. Así, Burgos y la Autònoma de Barcelona optan por la integración en la asignatura de Teoría e Historia de la Educación (UNIVERIDAD DE BURGOS, 2016 y UNIVERSITAT AUTÒNOMA DE BARCELONA, 2016). ${ }^{2}$ La Universitat Rovira i Virgili constituiría el caso extremo de práctica desaparición de la Historia de la Educación, pues si bien parece integrarla en la asignatura de Procesos y Contextos Educativos, lo cierto es que en su guía docente no hay ningún descriptor histórico entre sus contenidos y la competencia relativa al conocimiento histórico aparece únicamente vinculada a los exámenes, y no a ninguna otra actividad formativa como clases o trabajos. Sí que incluye por el contario dos resultados de aprendizaje relacionados con la historia, cuya consecución plantea la inquietante incógnita de si los aprendizajes relativos a la historia de la educación pueden adquirirse por ciencia infusa al margen de cualquier actividad formativa (UNIVERSIDAD ROVIRA Y VIRGILI, 2016).

Tabla 7. Universidades con sólo una asignatura o parte de ella. Fuente. Elaboración propia a partir de información oficial.

\begin{tabular}{|c|l|c|c|c|}
\hline Univ. & \multicolumn{1}{|c|}{ Nombre } & Tipo & Curso & Créd. \\
\hline UIB & Historia de la Educación & BAS & I & 6 \\
\hline USC & Historia de la Educación & OBL & 2 & 6 \\
\hline UAB & Teorías e Historia de la Educación & OBL & I & 4 \\
\hline UBU & Teoría e Historia de la Educación & BAS & I & 2 \\
\hline URV & Procesos y Contextos Educativos & BAS & I & $?$ \\
\hline
\end{tabular}

Como muestra la Tabla 7 , las cinco universidades que menos créditos otorgan a la Historia de la Educación lo hacen con la totalidad o parte de una sola asignatura en su

2 La carga que figura en la tabla se ha establecido a partir de la proporción de los contenidos correspondientes a historia en los totales de la asignatura. UNIVERSIDAD DE BURGOS (2016): Guía docente 2016-17. Teoría e Historia de la Educación. Teoría e Historia de la educación (GPED) 2016-17 (http://wwww. ubu.es/grado-en-pedagogia/informacion-basica/guias-docentes), consultado el 1 de abril de 2017 
plan de estudios. Todas ellas sitúan esta asignatura en el primer curso de carrera, excepto la Universidad de Santiago de Compostela que la coloca en el segundo. El escaso número de créditos más esta ubicación en el primer curso del plan de estudios (o segundo en Santiago) deja claro que estas universidades han optado por conferir a la Historia de la Educación un carácter introductorio en la formación de los/as futuros/as pedagogo/ as. La inclusión en los nuevos planes de otras materias de formación básica externas al núcleo de contenidos tradicionalmente incluidos en los planes de Pedagogía, como la economía o el derecho, amplifica este carácter introductorio. No cabe esperar que con esta ubicación y carga lectiva los/as pedagogos/as formados en estos planes consideren a la Historia de la Educación como un elemento sustancial de su formación, sino simplemente como un complemento formativo introductorio ajeno a sus competencias fundamentales.

La mayoría de las universidades españolas ha intentado paliar este carácter secundario e introductorio de la Historia de la Educación añadiendo una o más asignaturas a lo largo del programa de estudios. El grueso de ellas, ocho, lo ha hecho con una segunda asignatura, como muestra la Tabla 8. Las diferencias en el seno de este grupo radican en tres cuestiones: el curso en que esta segunda asignatura se coloca, su carácter obligatorio u optativo y el criterio seguido para la delimitación de sus contenidos.

Tabla 8. Universidades con más de una asignatura de Historia de la Educación. Fuente. Elaboración propia a partir de información oficial.

\begin{tabular}{|c|c|c|c|c|}
\hline Univ. & Nombre & Tipo & Curso & Créd. \\
\hline \multirow{2}{*}{ UdG } & Historia de la Educación y de la Pedagogía & OBL & 2 & 6 \\
\hline & Historia de la Educación en Cataluña & OPT & 2,3 y 4 & 3 \\
\hline \multirow[b]{2}{*}{ ULL } & Historia de la Educación & $\mathrm{OBL}$ & $\mathrm{I}$ & 6 \\
\hline & $\begin{array}{l}\text { Actividades de Integración 2:Análisis de los } \\
\text { Procesos Históricos y Políticas Educativas }\end{array}$ & OBL & $\mathrm{I}$ & 6 \\
\hline \multirow[b]{2}{*}{ UMA } & Historia de la Educación & $\mathrm{OBL}$ & $\mathrm{I}$ & 6 \\
\hline & $\begin{array}{l}\text { Historia de la Educación de la España } \\
\text { Contemporánea }\end{array}$ & $\mathrm{OBL}$ & 2 & 6 \\
\hline \multirow[b]{2}{*}{ UM } & Historia de la Educación & BAS & $\mathrm{I}$ & 6 \\
\hline & $\begin{array}{l}\text { Historia de los Sistemas Educativos } \\
\text { Contemporánea }\end{array}$ & $\mathrm{OBL}$ & 2 & 6 \\
\hline \multirow[b]{2}{*}{ UOV } & Historia de la Educación & BAS & $\mathrm{I}$ & 6 \\
\hline & $\begin{array}{l}\text { Historia de las Ideas Pedagógicas y del } \\
\text { Currículum }\end{array}$ & $\mathrm{OBL}$ & 3 & 6 \\
\hline \multirow[b]{2}{*}{ UPV } & Historia de la Educación & BAS & $\mathrm{I}$ & 6 \\
\hline & $\begin{array}{l}\text { Educación Contemporánea en Euskal } \\
\text { Herria }^{3}\end{array}$ & OPT & 4 & 6 \\
\hline \multirow{2}{*}{ USAL } & Historia de la Educación & BAS & I & 6 \\
\hline & Historia de la Educación en España & OPT & 4 & 6 \\
\hline \multirow{2}{*}{ UNED } & Historia de la Educación & BAS & $\mathrm{I}$ & 6 \\
\hline & Historia de la Educación Española & OBL & 2 & 6 \\
\hline
\end{tabular}

3 Aunque la palabra historia no aparezca en el título de esta asignatura, un análisis de los contenidos establecidos en su guía docente revela que se trata de una asignatura íntegramente dedicada a la historia de la educación (UNIVERSIDAD EL PAIS VASCO, 2016) 
La primera asignatura se ubica en todos los casos en el primer curso, exceptuando en Girona que la coloca en segundo. La segunda asignatura ha sido situada en segundo curso por cuatro de las nueve universidades, en tercero por una y en cuarto por dos. La Universidad de La Laguna es la única universidad española que coloca ambas asignaturas de historia en el mismo curso, y además las hace coincidir en el mismo cuatrimestre. En el caso de Girona, esta segunda asignatura puede cursarse en cualquier curso a excepción del primero.

Con respecto al carácter de esta segunda asignatura, la mayoría de las universidades, seis sobre nueve, la ha considerado obligatoria; mientras que tres la ofrecen como optativa.

Finalmente, cabe identificar tres criterios básicos para delimitar los contenidos de la segunda asignatura: temporal, geográfico y temático. Tres de las ocho universidades utiliza el criterio temporal para introducir una Historia de la Educación Contemporánea. Se trata de las universidades de La Laguna, Málaga y Murcia. Las universidades de Salamanca, UNED, Girona y el País Vasco, por su parte, utilizan el criterio geográfico para circunscribir esta segunda asignatura de Historia de la Educación a España en las dos primeras y a Cataluña y el País Vasco respectivamente en las últimas. Finalmente, la Universidad de Oviedo opta por el criterio temático y centra esta segunda asignatura de carácter histórico en las ideas pedagógicas y el currículo.

Tabla 9. Universidades con tres asignaturas de Historia de la Educación. Fuente. Elaboración propia a partir de información oficial.

\begin{tabular}{|c|c|c|c|c|}
\hline Univ. & Nombre & Tipo & Curso & Créd. \\
\hline \multirow{3}{*}{ UB } & Historia de la Educación & BAS & I & 6 \\
\hline & Historia de la Pedagogía Catalana & OPT & 2 & 3 \\
\hline & Memoria Histórica y Educación & OPT & 3 & 3 \\
\hline \multirow{3}{*}{ UCM } & $\begin{array}{l}\text { Historia y Corrientes Internacionales de la } \\
\text { Educación y la Cultura. }\end{array}$ & BAS & I & 6 \\
\hline & Historia del Pensamiento Pedagógico & OBL & 4 & 6 \\
\hline & Historia de la Educación de las Mujeres & OPT & 3 y 4 & 6 \\
\hline \multirow{3}{*}{ UGR } & Historia Social y Cultural de la Educación & BAS & I & 6 \\
\hline & $\begin{array}{l}\text { Historia del Sistema Educativo Español y sus } \\
\text { Instituciones }\end{array}$ & F.DISC & 2 & 6 \\
\hline & Historia de la Educación de las Mujeres & OPT & 3 & 6 \\
\hline \multirow{3}{*}{ US } & Historia de la Educación & BAS & I & 6 \\
\hline & Historia de la Educación Contemporánea & OBL & 2 & 6 \\
\hline & Desarrollo Educativo y Profesional de las Mujeres ${ }^{4}$ & OPT & 3 & 6 \\
\hline
\end{tabular}

Entre las cuatro universidades que ofrecen tres asignaturas de Historia de la Educación se distinguen claramente dos modelos: las universidades Complutense de Madrid, de Sevilla y de Granada, de un lado, y la de Barcelona, de otro. Las tres primeras

4 Aunque la palabra historia no aparezca en el título de esta asignatura, un análisis de los contenidos establecidos en su guía docente revela que se trata de una asignatura dedicada a la historia de la educación (UNIVERSIDAD DE SEVILLA, 2016) 
universidades incluyen 18 créditos de Historia de la Educación que distribuyen en tres asignaturas de seis créditos. En ambos casos, el carácter de estas asignaturas es el mismo: dos obligatorias y una optativa. Coinciden además estas tres universidades en la perspectiva de género para dedicar la asignatura optativa a la Historia de la Educación de las Mujeres. Difieren, sin embargo, en el criterio temático para delimitar los contenidos de la segunda asignatura y en su ubicación. La Universidad Complutense de Madrid dedica esta segunda historia obligatoria al pensamiento pedagógico (como Oviedo) y sitúa esta asignatura en cuarto. La Universidad de Granada, por su parte, opta por el sistema educativo español y sus instituciones y Sevilla por un planteamiento temporal más clásico. Ambas sitúan esta segunda asignatura obligatoria en el segundo curso.

La Universitat de Barcelona se diferencia de estas tres universidades en varios aspectos. En primer lugar, el número de créditos ofertados es sólo 12 en lugar de 18, pues la segunda y tercera asignaturas sólo tienen tres créditos. En segundo lugar, estas dos asignaturas son optativas en lugar de obligatorias como en las otras universidades. Por último, difiere también en el criterio utilizado para delimitar su contenido. De un lado, utiliza el criterio temático para introducir una asignatura dedicada a la memoria histórica; de otro, combina los criterios temático y geográfico para delimitar una Historia de la Pedagogía Catalana.

En todo caso, más allá de los criterios utilizados para delimitar las asignaturas, en estas cuatro universidades el alumnado recibe historia en los tres primeros cursos de su carrera, de manera obligatoria en los dos primeros (con la excepción de Barcelona) y optativa en el tercero. Estas universidades perfilan, por tanto, a la Historia de la Educación como un elemento constitutivo del currículum formativo de la Pedagogía y no como una simple materia introductoria a la manera de la mayoría de universidades españolas.

Tabla 10. Universidades con cuatro asignaturas de Historia de la Educación. Fuente. Elaboración propia a partir de información oficial.

\begin{tabular}{|c|l|c|c|c|}
\hline Univ. & \multicolumn{1}{|c|}{ Nombre } & Tipo & Créd. & Curso \\
\hline \multirow{4}{*}{ UV } & Fundamentos Históricos de la Educación & BAS & I & 6 \\
\cline { 2 - 5 } & Historia de la Educación en España & OBL & 3 & 6 \\
\cline { 2 - 5 } & Historia de la Educación de las Mujeres & OPT & 4 & 4,5 \\
\cline { 2 - 5 } & Historia Política de la Educación & OPT & 4 & 4,5 \\
\hline
\end{tabular}

La Universitat de València, por su parte, refuerza esta importancia de la Historia de la Educación en su plan formativo y se sitúa a la cabeza de las universidades españolas, no sólo por el número de créditos dedicados a historia de la educación (21), sino también por su distribución en 4 asignaturas. Para la delimitación de la segunda asignatura obligatoria esta universidad se decanta por el criterio geográfico, como Granada, País Vasco, UNED y Salamanca. Estas dos asignaturas obligatorias se ven complementadas con dos asignaturas optativas: una Historia de la Educación de las Mujeres y una Historia Política de la Educación. Coincide, por tanto, con Granada, Sevilla y Complutense de Madrid en la perspectiva de género para crear la tercera asignatura y añade una perspectiva única (la política) para la cuarta. 


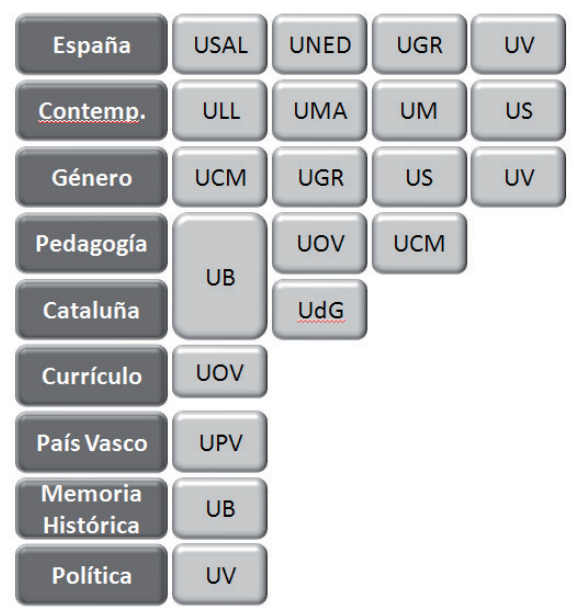

Diagrama 3. Otras asignaturas. Fuente. Elaboración propia a partir de información oficial.

El resultado de la combinación de los diferentes criterios para la delimitación de otras asignaturas aparte de la Historia de la Educación ofrece una oferta mucho más limitada que el caso de las licenciaturas. El criterio geográfico da lugar a una asignatura acotada a España en cuatro universidades (Salamanca, Granada, Valencia y UNED) y a las respectivas comunidades en Barcelona, Girona y el País Vasco. El criterio temporal genera historias contemporáneas de la educación en cuatro universidades (La Laguna, Málaga, Murcia y Sevilla). Cuatro son también las universidades que utilizan el criterio de género (Granada, Complutense de Madrid, Sevilla y Valencia). La novedad más destacable de esta oferta es la aparición en tres universidades (Barcelona, Oviedo y Complutense de Madrid) de una Historia de la Ideas Pedagógicas, una dimensión inexistente en los planes anteriores. Finalmente, la Universidad de Oviedo mantiene la Historia del Currículo, la de Valencia la Historia Política de la Educación y Barcelona introduce la memoria histórica.

\section{Recapitulación:}

Cinco universidades españolas (27\%) ofrecen seis créditos o menos de Historia de la Educación en sus planes de estudios de Pedagogía (Illes Balears, Santiago de Compostela, Autónoma de Barcelona, Burgos y Rovira i Virgili, en orden decreciente).

Ocho universidades, el 44 \%, ofrecen 12 créditos (Barcelona, La Laguna, Málaga, Murcia, Oviedo, País Vasco, Salamanca y UNED).

Sólo cuatro universidades, el $22 \%$, ofrecen más de 12 créditos: Sevilla, Granada y Complutense de Madrid con 18 créditos y Valencia con 21.

Las universidades que sólo ofrecen seis créditos, o menos, incluyen en su plan de estudios una única asignatura que se sitúa en primer curso, con la excepción de la Universidad de Santiago de Compostela que la coloca en segundo.

Las universidades que ofrecen 12 créditos lo hacen con dos asignaturas, mayoritariamente obligatorias, con la excepción de Barcelona, País Vasco y Salamanca que incluyen una o varias optativas.

La Universidad Complutense de Madrid y las de Sevilla y Granada ofrecen 18 créditos en tres asignaturas: dos obligatorias y una optativa.

La Universitat de València se sitúa en la cabeza de las universidades españolas tanto por número de créditos de Historia de la Educación ofrecidos (21) como por asignaturas: dos obligatorias y dos optativas.

A pesar de esta variabilidad en la oferta, las universidades españolas se dividen únicamente en dos grupos con respecto a los créditos obligatorios. La mitad establece 12, la otra mitad sólo seis. 
El criterio seguido para la delimitación de la segunda asignatura, mayoritariamente obligatoria, es:

- de género en las universidades Complutense de Madrid, Sevilla, Granada y València.

- temporal en las universidades de La Laguna, Málaga, Murcia y Sevilla, dando lugar a Historias Contemporáneas de la Educación.

- geográfico en Granada, Salamanca, UNED, País Vasco, Girona y Valencia, dando lugar a Historias de la Educación en España, Cataluña y País Vasco.

- temático en Barcelona, Complutense de Madrid y Oviedo, dando lugar a asignaturas de Historia del Pensamiento Pedagógico.

\section{Un campo en extinción}

La comparación de los planes de estudios de las últimas licenciaturas y los nuevos grados muestra una drástica reducción de los créditos de Historia de la Educación en los títulos de Pedagogía. Esta reducción es de casi la mitad en la obligatoriedad y de nada menos que dos tercios en la optatividad. Se trata, por tanto, de un retroceso radical.

Tabla 11. Comparación de los créditos de Historia de la Educación en las licenciaturas y los grados de Pedagogía. Fuente. Elaboración propia a partir de información oficial.

\begin{tabular}{|c|c|c|c|c|c|}
\hline \multicolumn{3}{|c|}{ Licenciatura en Pedagogía } & \multicolumn{3}{|c|}{ Grado en Pedagogía } \\
\hline Univ. & Oblig & Total & Univ. & Oblig & Total \\
\hline UV & 21 & 55,5 & UV & 12 & 21 \\
\hline ULL & 19,5 & 33 & UCM & 12 & 18 \\
\hline UB & 18 & 48 & UGR & 12 & 18 \\
\hline UOV & 18 & 27 & US & 12 & 18 \\
\hline UCM & 18 & 24 & ULL & 12 & 12 \\
\hline US & 17 & 26 & UMA & 12 & 12 \\
\hline UMA & 16,5 & 34,5 & UM & 12 & 12 \\
\hline UGR & 15 & 33 & UOV & 12 & 12 \\
\hline UM & 15 & 28,5 & UNED & 12 & 12 \\
\hline UPV & 15 & 27 & UB & 6 & 12 \\
\hline UIB & 15 & 27 & UPV & 6 & 12 \\
\hline UNED & 15 & 25 & USAL & 6 & 12 \\
\hline USAL & 15 & 24 & UdG & 6 & 9 \\
\hline URV & 13,5 & 31,5 & UIB & 6 & 6 \\
\hline USC & 9 & 13,5 & USC & 6 & 6 \\
\hline UAB & 8 & 15 & UAB & 4 & 4 \\
\hline UdG* & 6 & 15 & UBU & 3 & 2 \\
\hline UBU* & 0 & 24 & URV & $i$ & $?$ \\
\hline MEDIA $^{\&}$ & $|5,7|$ & 30,09 & MEDIA & 8,8 & II \\
\hline \multicolumn{6}{|c|}{ * Titulación de segundo ciclo } \\
\hline \& Sólo titulac & npletas & & & & \\
\hline
\end{tabular}


No obstante, algunos cambios producidos en el diseño de los títulos de grado como la reducción del número de créditos de los grados y la radical reducción de la optatividad pueden distorsionar un tanto esta primera imagen. Por ello es necesario acotar los elementos a comparar.

Tomando en consideración únicamente la obligatoriedad, obtenemos que mientras la media de la carga global de los títulos se ha reducido en algo más de un $20 \%$, la de la carga obligatoria de Historia de la Educación lo ha hecho en un $44 \%$, es decir, en más del doble. Para mantener la proporcionalidad, la carga obligatoria de Historia de la Educación en los grados debería superar los 8,8 créditos actuales para situarse en torno a los 12. De hecho, así parecen haberlo entendido la mayoría de las universidades que se ajusta a esa cifra en su obligatoriedad.

La reducción de la optatividad ha sido mucho más radical. Sólo siete universidades completan su obligatoriedad con asignaturas optativas; cinco con una y dos con dos. No obstante, la introducción de optatividad ya no es un indicador de la fortaleza de la especialidad, pues depende de la dimensión de la obligatoriedad a la que completan, si 6, 12 o 18 créditos. Dentro del grupo de las universidades que sitúan su oferta en los 12 créditos, la mayoría ha apostado por la obligatoriedad de todos ellos, mientras que tres combinan una obligatoriedad mínima de 6 créditos con la misma carga de optatividad. En todo caso, tomando en cuenta la optatividad, el peso de la historia de la educación en los nuevos grados de Pedagogía ha quedado reducida a un tercio de su peso en las antiguas licenciaturas.

En términos generales, los 12 créditos que la mayoría de las universidades reserva para la Historia de la Educación suponen un $5 \%$ de la carga total del título, un porcentaje que se incrementa hasta el 7,5 \% en Granada, Sevilla y la Complutense y hasta un máximo del 8,75 \% en Valencia, en caso de que el alumnado cursara todas las asignaturas optativas. Por contraste, los 30 créditos obligatorios y optativos de las licenciaturas suponían un $10 \%$ de la carga del título.

Con respecto a las posiciones relativas de las universidades no se detectan como norma cambios dramáticos. Cuatro universidades repiten en el grupo de cola: Autònoma de Barcelona, Santiago de Compostela, Burgos y Girona. Las dos primeras ya ocupaban las últimas posiciones en las licenciaturas y el paso a la titulación completa en los últimos casos no se ha traducido en un aumento de los contenidos de historia. En el otro extremo, el liderazgo sigue correspondiendo a la Universitat de València, seguida ahora por la Complutense de Madrid, Granada y Sevilla, en detrimento de la posición anterior de La Laguna y sobre todo de Barcelona, que cae en picado desde la tercera a la décima posición.

Además de empobrecer el rico abanico de las licenciaturas, la drástica reducción de la oferta de asignaturas históricas revela algunos cambios significativos en los criterios priorizados por las universidades para la creación de asignaturas. El cambio más importante se ha producido en las historias acotadas geográficamente que se han reducido de 21 a tan sólo seis. Por el contrario, han aparecido dos nuevos criterios: el temporal en cuatro casos (La Laguna, Málaga, Murcia y Sevilla) y la historia del pensamiento pedagógico en tres (Barcelona, Complutense de Madrid y Oviedo). Finalmente, en contra de lo que pudiera parecer a primera vista, la perspectiva de género en Historia de la Educación ha conseguido minimizar los daños en una coyuntura tan adversa como fue la última reforma con asignaturas en cuatro universidades frente a las cinco anteriores. Las universidades Granada, Sevilla y Valencia, que tradicionalmente han liderado 
la investigación en este campo, mantienen sus asignaturas, mientras que la perspectiva de género desaparece en Murcia y la Rovira y Virgili. En contra de esta tendencia, se ha incorporado en la Complutense de Madrid, que cuenta con un notable grupo de investigación en el campo.

En conclusión, pues, en el tránsito de las licenciaturas a los nuevos grados, la presencia de la Historia de la Educación en los planes de estudio de Pedagogía se ha visto reducida a la mitad en la obligatoriedad y a un tercio en la oferta total incluyendo la optatividad. Se trata a todas luces de un duro golpe que derriba lo poco que quedaba de la antigua posición central en la formación de los futuros profesionales de la Pedagogía. Un paso más significaría la práctica erradicación, y no es poco común la sensación entre los profesionales del campo de que podía haber sido todavía peor y que por esta vez se ha conjurado ese peligro. No obstante este alivio coyuntural, no debe ocultar que esta escasa presencia se circunscribe a los primeros cursos, de tal manera que para el futuro profesional de la Pedagogía la historia ya no es más que una breve contextualización inicial, más o menos curiosa y atractiva, pero completamente ajena a las cuestiones claves que se barajan en su campo disciplinar. Únicamente, en las universidades Complutense de Madrid, de Granada, de Sevilla y de València la presencia de asignaturas históricas de manera continuada y en los últimos cursos de carrera rompe con esta lamentable situación y apunta a la consideración de la Historia de la Educación como una línea de investigación solvente y con credibilidad en las facultades de educación. Así, pues, la Historia de la Educación es hoy un campo en extinción en su antiguo feudo de las titulaciones de Pedagogía.

\section{Referencias Bibliográficas}

ÁLVAREZ GONZÁLEZ, Y. (2012): La historia de la educación en los nuevos grados de Pedagogía de las universidades públicas españolas, en J.M. HERNÁNDEZ, (Ed.), Formación de Élites y Educación Superior en Iberoamérica (SS. XVI y XXI), II, pp. 389- 397 (Salamanca, Hergar).

CASPARD, P. \& ROGERS, R. (2012): The history of education in France: a laboriously useless science?, en J.E. LARSEN, (ed.), Knowledge, Politics and the History of Education, pp. 73-85 (Copenhague: LIT-Verlag).

DEPAEPE, M. (2006): Vieja y nueva memoria de la educación (Barcelona, Octaedro).

FITZGERALD, T. (2013): Contemporary Timelines and Strategic Possibilities in the History of Education. International Journal for the Historiography of Education - Bildungsgeschichte, 2, pp. 240-241.

FUCHS, E. (2010): Historische Bildungsforschung in internationaler Perspektive: Geschichte - Stand - Perspektiven, Zeitschrift für Pädagogik, 56, 5, pp. 703-724.

GRANA GIL, I. (2012): La Historia de la Educación en los Nuevos Planes de Estudios, $X X$ Jornades d>història de l'educació: cohesió social i educación, pp.531-542, (Girona, Universitat de Girona).

GUEREÑA, J. L., RUIZ BERRIO, J., TIANA FERRER, A. (Eds.) (2010): Nuevas miradas historiográficas sobre la educación en los siglos XIX y XX (Madrid, IFIIE, Ministerio de Educación). 
ISCHE (International Standing Conference of the History of Education) (2017): Standing Working Groups (http://www.ische.org/about-ische/standing-working-groups/), consultado el 1 de abril de 2017.

LE CAM, J.-L. (2013): L'histoire de l'éducation : discipline de recherche historique ou science auxiliaire de l'action pédagogique? Les leçons d'une comparaison francoallemande, Histoire de l'éducation, 137, pp. 93-123. (link is external).

MAINER BAQUE, J. (2009): La forja de un campo profesional. Pedagogía y didáctica de las ciencias sociales en España (1900-1970) (Madrid, CSIC).

MCCULLOCH, G. (2011): The Struggle for the History of Education (Abingdon, Routledge).

OSTERWALDER, F. (2012): Bildungsprogramme, Bildungsreformen und die longue durée in der pädagogische Geschichtsschreibung, International Journal for the Historiography of Education - Bildungsgeschichte, 2, pp. 206-220.

RICHARDSON, W. (2000): History, education and the audience, en CROOK, D. y ALDRICH, R., History of Education for the Twenty-First Century, pp. 17-35 (Londres, Institute of education).

SANCHIDRIÁN BLANCO, M. del C. (2016): Tesis de Historia de la Educación en la base de datos TESEO (España, 2000-2010), Espacio, Tiempo y Educación, 3, 1.

SANCHIDRIÁN, C. (2013): La historia cultural de la educación: entre cambios y continuidades (Málaga, Universidad de Málaga).

UNIVERSIDAD DE BURGOS (2016): Guía docente 2016-17. Teoría e Historia de la Educación. Teoría e Historia de la educación (GPED) 2016-17 (http://wwww.ubu. es/grado-en-pedagogia/informacion-basica/guias-docentes), consultado el 1 de abril de 2017

UNIVERSIDAD DE SEVILLA (2016): Desarrollo Educativo y Profesional de las Mujeres (Grado de Pedagogía) http://www.us.es/estudios/grados/plan_174/ asignatura_1740038, consultado del 1 de abril de 2017.

UNIVERSIDAD DEL PAIS VASCO (2016): 26317. Educación Contemporánea en Euskal Herria (http://www.ehu.eus/es/web/vicer.grado-innovacion/ aurtengo-gradu-guztiak?p_p_id=upvehuapp_WAR_upvehuappportlet\&p_p_ state $=$ normal $\& p \_p \_m o d e=v i e w \& p \_p \_c o l \_i d=c o l u m n-2 \& p \_p \_c o l \_p o s=0 \& p \_p \_$

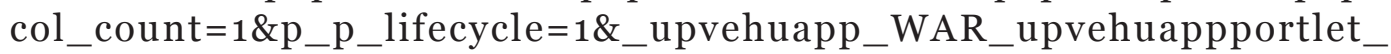
action $=$ redirectAction\&reu $=/ \mathrm{pls} /$ entrada/plewo040.htm_asignatura_next?p_ sesion $=\& p \_c o d \_i d i o m a=C A S \& p \_e n \_p o r t a l=N \& p \_c o d \_c e n t r o=230 \& p \_c o d \_$ plan $=$ GPEDAG2o\&p_anyoAcad $=$ act $\&$ p_pestanya $=3 \& p \_m e n u=g u i a \& p \_c o d \_$ asig $=26317 \& p \_c i c l o=X \& p \_c u r s o=4 \& p \_d p t o=\& p \_v e n g o \_d e=a s i g \_c u r s o s \& p \_$ centro_ori=230\&p_plan_ori=GPEDAG20), consultado el 1 de abril de 2017).

UNIVERSIDAD ROVIRA Y VIRGILI (2016): Guía docente 2016-17. Grado de Pedagogía, 2009. Procesos y Contextos Educativos (https://moodle.urv.cat/docnet/guia_ docent $/$ index.php? centre $=1^{1} \&$ ensenyament $=1121 \&$ assignatura $=11214002 \&$ any academic $=2016 \_17$ \&idioma $=$ cast\&any_academic $\left.=2016 \_17\right)$, consultado del $1 \mathrm{de}$ abril de 2017. 
UNIVERSITAT AUTÔNOMA DE BARCELONA (2016): Teoría e Historia de la Educación 2016-17 (http://www.uab.cat/guiesdocents/2016-17/g101662a2016-17iSPA.pdf), consultado el 1 de abril de 2017.

VIÑAO FRAGO, A. (2008): La escuela y la escolaridad como objetos históricos. Facetas y problemas de la Historia de la Educación, en J. MAINER, (coord.), Pensar críticamente la educación escolar, pp. 83-118 (Zaragoza, Prensas Universitarias de Zaragoza).

VIÑAO FRAGO, A. (2016): La Historia de la Educación como disciplina y campo de investigación: viejas y nuevas cuestiones, Espacio, Tiempo y Educación, 3, 1, pp. 21-42.

\section{Fuentes oficiales}

\section{Licenciaturas:}

Universidad Complutense de Madrid: BOE 14 de noviembre de 2000

Universidad de Burgos: BOE 23 de octubre de 1998

Universidad de Granada: BOE 10 de febrero 2001

Universidad de La Laguna: BOE 3 de febrero de 2000

Universidad de Málaga: BOE 2 de abril de 1999

Universidad de Murcia: BOE 13 de marzo de 2001

Universidad de Oviedo: BOE 30 de agosto de 2000

Universidad de Salamanca: BOE 23 de agosto de 2000

Universidad de Sevilla: BOE 20 de julio de 1998

Universidad del País Vasco: BOE 14 de mayo de 1999

Universidad Nacional de Educación a Distancia: BOE o3 de diciembre de 1999

Universidade de Santiago de Compostela: BOE 24 de agosto de 2001

Universitat Autònoma de Barcelona: BOE 16 de octubre de 1993

Universitat de Barcelona: BOE, 1 de diciembre de 2000

Universitat de Girona: BOE 10 de febrero de 2000

Universitat de les Illes Balears: BOE, 17 de diciembre de 1997

Universitat de València: BOE 16 de agosto de 2000

Universitat Rovira i Virgili: BOE 25 de noviembre de 1993

\section{Grados:}

Universidad Complutense de Madrid: BOE 29 de junio de 2010

Universidad de Burgos: BOE 7 de marzo de 2011

Universidad de Granada: BOE 30 de mayo de 2011

Universidad de La Laguna: BOE 22 de abril de 2011

Universidad de Málaga: BOE 28 de mayo de 2012

Universidad de Murcia: BOE 15 de julio de 2010

Universidad de Oviedo: BOE 12 de julio de 2011

Universidad de Salamanca: BOE 19 de mayo de 2011

Universitat de València: BOE 30 de diciembre de 2011

Universidad de Sevilla: BOE 20 de enero de 2011

Universidad del País Vasco: BOE 4 de febrero de 2011

Universidad Nacional de Educación a Distancia: BOE 17 de octubre de 2011

Universidade de Santiago de Compostela: BOE 5 de marzo de 2010

Universitat Autònoma de Barcelona: 30 de octubre de 2013

Universitat de Barcelona: BOE 10 de abril de 2012

Universitat de Girona: BOE 30 de enero de 2015

Universitat de les Illes Balears: BOE 19 de julio de 2010

Universitat Rovira i Virgili: BOE 11 de agosto de 2011 


\section{Anexo 1: Abreviaturas utilizadas}

\begin{tabular}{|l|l|}
\hline \multicolumn{1}{|c|}{ Siglas } & \multicolumn{1}{c|}{ Universidad } \\
\hline UAB & Universitat Autònoma de Barcelona \\
\hline UB & Universitat de Barcelona \\
\hline UBU & Universidad de Burgos \\
\hline UCM & Universidad Complutense de Madrid \\
\hline UdG & Universitat de Girona \\
\hline UGR & Universidad de Granada \\
\hline UIB & Universitat de les Illes Balears \\
\hline ULL & Universidad de La Laguna \\
\hline UM & Universidad de Murcia \\
\hline UMA & Universidad de Málaga \\
\hline UNED & Universidad Nacional de Educación a Distancia \\
\hline UOV & Universidad de Oviedo \\
\hline UPV & Universidad del País Vasco \\
\hline URV & Universitat Rovira iVirgili \\
\hline US & Universidad de Sevilla \\
\hline USAL & Universidad de Salamanca \\
\hline USC & Universidad de Santiago de Compostela \\
\hline UV & Universitat de València \\
\hline
\end{tabular}

